\title{
Root cause investigation and prevention of dynamic fluctuations in angular-resolved torque measurements of high pressure fuel pumps
}

\author{
Simon Schindler ${ }^{1} \cdot$ Thorsten Hergemöller $^{1}$ - Olaf Tödter ${ }^{2} \cdot$ Thomas Koch $^{2}$
}

Received: 12 April 2017/Accepted: 26 July 2017/Published online: 17 August 2017

(C) Springer International Publishing AG 2017

\begin{abstract}
In terms of better fuel mixture preparation, both finer atomization and higher system pressure are indispensable. These objectives provoke high mechanical loads on modern injection systems. In particular, the driving torque of the high pressure pump is proportionally linked to the system pressure within the pump cylinder. This article focuses the challenge of measuring angular-resolved torque fluctuations induced by the high pressure pump on a component test bench. After describing a systematic measurement failure consisting of unexpected dynamic proportions within the torque signal, the disturbance is analyzed at steady-state and transient operating conditions of the test bench. Consequently, a method for detecting and analyzing the same within the measurement chain of the signal is suggested. Based on the identification of the root cause of the dynamic fluctuations, enhancement proposals are discussed, realized and validated.
\end{abstract}

Keywords Dynamic torque - Vibration - Resonance · Driveline $\cdot \mathrm{NVH}$

$\begin{array}{ll}\text { Abbreviations } \\ \text { RPM } & \text { Rotations per minute } \\ \text { SDOF } & \text { Single degree of freedom } \\ \text { FTA } & \text { Fault tree analysis }\end{array}$

Simon Schindler

simon.schindler13@gmail.com

Daimler AG, Stuttgart, Germany

2 IFKM, Karlsruhe Institute of Technology, Karlsruhe, Germany

\section{Introduction}

The request for both constantly decreasing emission levels and reduction of the fuel consumption leads to highest requirements for modern drive concepts with combustion engines. Here, parameters like valve lift and valve timing play a vital role and are regulated by the camshaft and its adjustment. In state-of-the-art direct injecting Otto engines, the camshaft impels the high pressure fuel pump at the same time. Due to increasing system pressure, dynamic torque peaks evoked by the high pressure pump rise continuously. These cause dynamic stress fluctuations of the camshaft that can influence the valve dynamics tremendously. In contrast to that, the high pressure pump of state-of-the-art Diesel engines is frequently driven by an auxiliary shaft or is integrated within the chain drive of the engine because of the higher system pressure. In both cases, the dynamic torque peaks influence the surroundings negatively through dynamic mechanical load fluctuations. The measurements and analysis within this article are based on experiments of high pressure pumps for Otto engines.

The analysis of the torque applied to the high pressure pump is conventionally performed on engine test benches and represents an element in loss sharing experiments. Automotive component test benches frequently enable the measurement of an average torque value for the pump generated by hardware-based digital filtering. In turn, dynamic proportions are neglected and not taken into account $[1,2]$.

\section{Laboratory setup}

\subsection{Test stand environment}

The configuration of the test bench for the evaluation of high pressure pumps for direct fuel injection is depicted in 


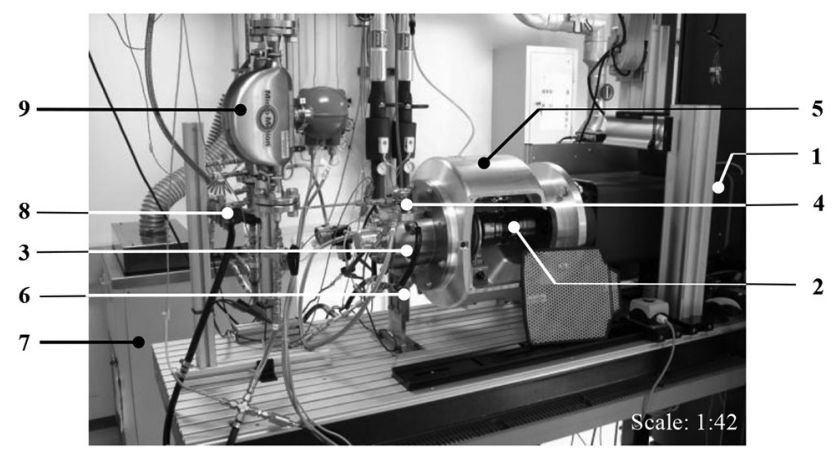

Fig. 1 Arrangement of the test laboratory (ㄷ Daimler AG)

Fig. 1. The setup consists of an asynchronous machine (1) as power unit of the driveline. The electric motor is followed by a shaft coupling and a torque measurement system (2). At the output shaft (3), a multi-lobe cam drives the actual pump (4). The driveline is enclosed by a cylindrical aluminum housing (5) and retained on the measuring table by a vertical support beam (6). The lubricant for the externally lubricated roller tappet contact is delivered by an oil pump and conditioning unit, located underneath the measuring table. The high pressure pump is included into the hydraulic circuit, consisting of a conditioned fuel tank (7), an auxiliary pump, the common rail (8) and the flowmeter device (9) to measure mass flow based on the Coriolis effect. Both oil and fuel circuit are equipped with various pressure transducers and temperature sensors.

The design of the test bench aims at the evaluation of diverse quality criteria such as volumetric and mechanic efficiency, low and high pressure oscillations, heat release or power consumption during standardized drive cycles.

\subsection{Integration of the specimen into the test setup}

The mechanical implementation of the high pressure fuel generators for gasoline engines both into the engine and into the test setup is schematically illustrated in Fig. 2. The arrangement is driven by the camshaft or rather its analogon on the automotive component test bench. To stabilize the rail pressure level and diminish the impact of pressure oscillation events on the injection rate, the shaft is equipped with a multi-lobe cam (1) for the propulsion of the pump. Four-cylinder engines commonly feature four lobes, as depicted in Fig. 2, to synchronize injection and pump events, enabling the neutralization of the pressure peaks within the common rail. The rotatory movement is translated into translatory lift of the piston (3) by an externally lubricated roller tappet (2). To prevent the roller tappet from taking off, the piston is supported by an accordingly designed coil spring. Through the high pressure within the cylinder of the pump, piston, roller tappet and cam are subjected to tremendous mechanical forces. The pump

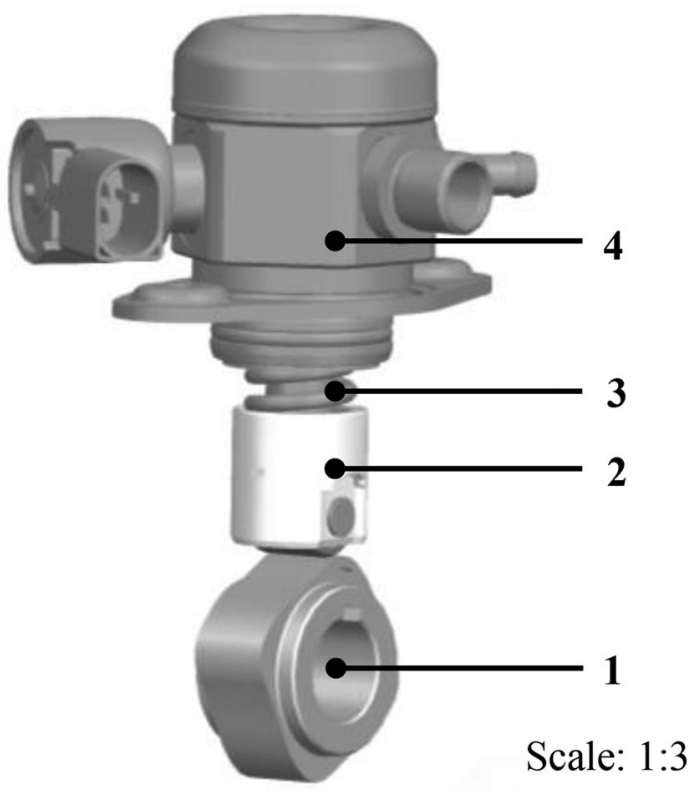

Fig. 2 Roller tappet-cam contact (C Daimler AG)

body (4) is assembled to the cylinder head or rather to the appropriate flange on the component test bench.

\subsection{Measurement of the torque induced by the pump}

The measurement of the torque induced by the pump onto the camshaft is performed contactlessly by a stator-rotor system. Typically, the quantification of torque is based on the measurement of torsion as indirect measured value. The measurement principle is based on the elongation and compression of multiple strain gages and their consequent change in electric resistance. To achieve a high sensitivity, the measurement rotor generally has a high overall torsional stiffness. However, areas that are equipped with strain gages are marked by low torsional stiffness. Furthermore, the strain gages possess resistance wire lined in a meander shape to increase their length within the smallest possible area. The rotor is incorporated into the driveline of the test setup as close to the high pressure pump as possible. Hence, damping within the shaft and influences of other rotary parts on the measured torque are diminished $[3,4]$.

\section{Evaluation of the dynamic torque signal}

\subsection{Problem statement}

Initial investigations of the dynamic and unfiltered torque signal contain an unexpected dynamic proportion existing for all operation conditions of the system. Each steady-state 
Fig. 3 Torque signal at 2700 RPM, 400 bar, $30 \mathrm{~kg} / \mathrm{h}$ (C) Daimler AG)

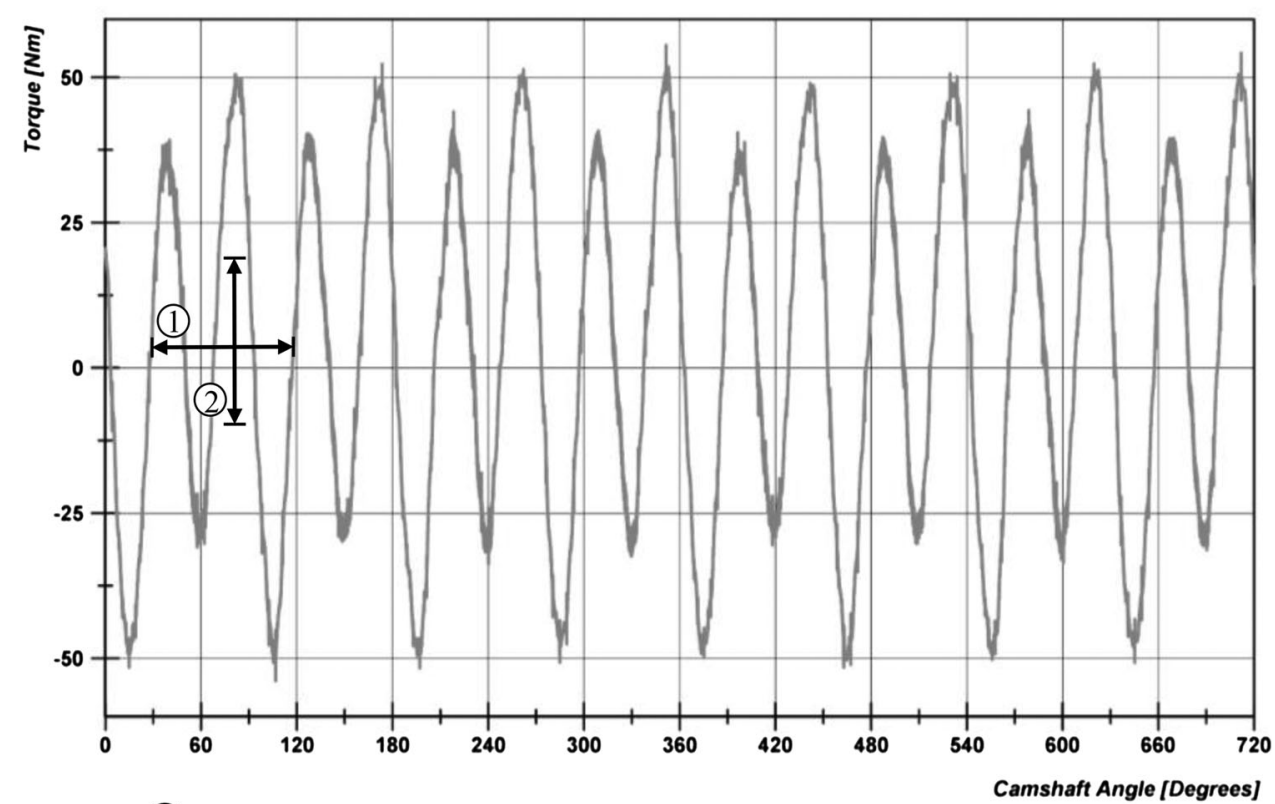

(1) Cycle duration for a four-lobe cam

(2) Mathematically estimated working stroke of the torque operating point is described by the parameter tribe of rotation speed $n$, pressure $p$ and mass flow $\dot{m}$. The dynamic proportion is superimposed onto the dynamic torque signal induced by the multi-lobe cam profile. Depending on the parameter tribe $(n, p, \dot{m})$, the measured torque signal is impaired significantly. Figure 3 paradigmatically shows the measurement for two full rotations of the four-lobe cam at the steady-state operating point (2700 RPM, 400 bar, $30 \mathrm{~kg} / \mathrm{h}$ ). It is obvious that the signal barely shows the eight expected torque cycles due to eight pump strokes within two full rotations. However, the measurement contains a dynamic proportion of unknown origin. The expected cycle duration and working cycle for the torque oscillation are schematically indicated within the graph.

\subsection{Systematic root cause investigation and identification of the dynamic measurement failure}

To systematize the root cause possibilities for the characteristic measurement failure, a fault tree analysis is performed $[5,6]$.

In the course of this type of deductive failure analysis, the possible root causes are divided into an electrical, a mechanical and a hydraulic branch. The electrical branch connotes that the dynamic fluctuations of torque are based on voltage fluctuations superimposed to the real signal and generated at a distinctive point within the measurement chain. Secondly, an electrically intact measurement chain can acquire a mechanical effect within the driveline and its

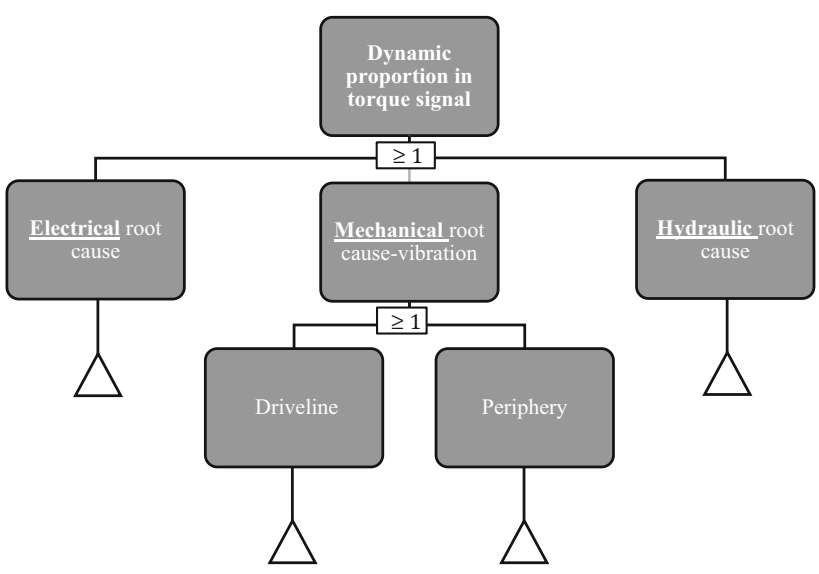

Fig. 4 Initial event and top part of fault tree diagram (ㄷ Daimler AG)

periphery during the system in operation. The third main branch of the fault tree is characterized by the hypothesis of a hydraulic source of the torque fluctuations. These are predominantly pressure oscillations on the low and high pressure side of the pump impairing the signal.

To fractionally execute the fault tree analysis (FTA) during the root cause investigation, exclusion experiments are performed. These prove or refute the requirements for the existence of sections or subsections linked by Boolean operators within the tree structure. Figure 4 descriptively illustrates the initial event and the top part of the diagram.

For the analysis of the electrical branch, the measurement chain is analyzed from the monitor display over the 
third party data acquisition software to the strain gages within the rotor of the torque measurement system. Here, analysis tools such as a galvanically decoupled oscilloscope are employed. As long as the dynamic torque proportion can be proved at a certain point within the measurement chain, all preceding components can be excluded from the investigation.

Due to the lack of a signal flow and the complex dynamic behavior and correlation of the structure as a whole and its components, the mechanical branch is characterized by multiple OR gates. In turn, this branch requires a comparably larger amount of exclusion experiments. Effectively, the modal behavior of the driveline and its periphery can be tuned by its mass, stiffness or damping. These effects are being taken advantage of to provoke a possible effect in the measured signal.

The hydraulic branch of the FTA is analyzed by reference pressure signals and correlates the same with the measured torque within the driveline. Here, the indicated pressure within the pump cylinder is measured, as it is directly associated with the dynamic torque by the mechanical transmission chain of the piston and the roller tappet. If the indicated pressure signal shows the parasitic dynamic proportion, it may also appear within the measured pressure signal on either the low or high pressure side of the pump.

The described analysis procedure detects potential root causes for the measurement failure and eventual scientific insights about the reason itself. In summary, the oscillation stays within the measured signal despite all attempts and hardware swaps within the electrical signal flow. Additionally, the comparison with the measured pressure does not reveal any relation with the detected dynamic torque proportion neither within the low and high pressure signals, nor within the indicated cylinder pressure. Eventually and over a course of multiple structural changes within the periphery, the signal can be tremendously influenced by changing the rotary mass of the driveline. This gives proof to the fact that the cause of the measurement failure is rooted in the modal characteristics of the powertrain of the test facility.

Concerning eigenmodes of the driveline itself, axial modes, bending modes or torsional modes can be excited by the system and influence the measured torque. Exclusion of certain deformation theories can be achieved by performing a numerical modal analysis of the system on the base of a flexible multibody model. However, an experimental approach is chosen for the present system by exciting the driveline in a broad frequency band. This is achieved by holding the rest position with the use of the speed regulator. Consequently, the regulator keeps the shaft in between one of the 1024 increments of the speed regulation with a frequency of $\sim 23 \mathrm{~Hz}$. This regulation excites the structure in a broad frequency band containing the resonant frequency. The response of the driveline is measured by triaxial accelerometers which are attached at different locations along the driveline. The animation of operational deflection shapes $[7,8]$ at this operating condition shows, that the motion of the shafts at resonant frequency is intensively characterized by torsion. These considerations lead to the hypothesis that the measured signal is deteriorated by the excitation of the first torsional resonance of the driveline as a whole. The hypothesis is confirmed by simulating the torsional resonance of the driveline by the use of a rigid body model and establishing the equations of motion according to Lagrange [9]. In this analytical simplification of the driveline, the first torsional resonance is located at $363.7 \mathrm{~Hz}$.

Transient information about the vibrational characteristics of the measured torque signal is given by performing a frequency analysis of a rotation speed sweep and plotting the results in a Campbell diagram, as depicted in Fig. 5.

The power spectral density diagram of the torque signal illustrates both desired and undesired dynamic proportions. Obviously, dynamic proportions carrying the resonant frequency of $f_{\text {res }} \approx 375 \mathrm{~Hz}$ are excited throughout the whole rotational speed range. Additionally, the 4th rotation order of the exciting four-lobe cam dominates the vibrational characteristics. Moreover, the diagram shows that the resonance is permanently excited by multiples of the 4th rotation order of the camshaft. With one of the harmonic rotation orders crossing the resonant frequency, the signal in the time domain barely shows its 4 th order origin. An example is stated by the 8th order exciting frequencies in the resonant range at 2700 RPM in Fig. 3. Supplementary, it is obvious that the oscillation of the torque signal is significantly attenuated roughly above $\sim 550 \mathrm{~Hz}$. This observation connotes that the system behaves similar to a single degree of freedom (SDOF) oscillator within a frequency range $\leq 1 \mathrm{kHz}$. Hence, both the torque applied to the pump, represented by the multiples of the 4th rotation order and the superimposed, undesired vibrational proportion are attenuated above a frequency of $\sqrt{2} \cdot f_{\text {res }} \approx 530 \mathrm{~Hz}$.

For frequencies below resonance, the signal is amplified, depending on the damping ratio within the system.

\subsection{Enhancement of the system}

Opportunities for the improvement of the system can be categorized into the treatment of the dynamic input on the one hand and possibilities which focus the enhancement of the dynamic output on the other hand. Generally, vibration management possibilities do not equal in efficiency. Figure 6 overviews a hierarchical arrangement of the methodology in relation to the present system. 
Fig. 5 Campbell diagram of measured torque signal (C) Daimler AG)

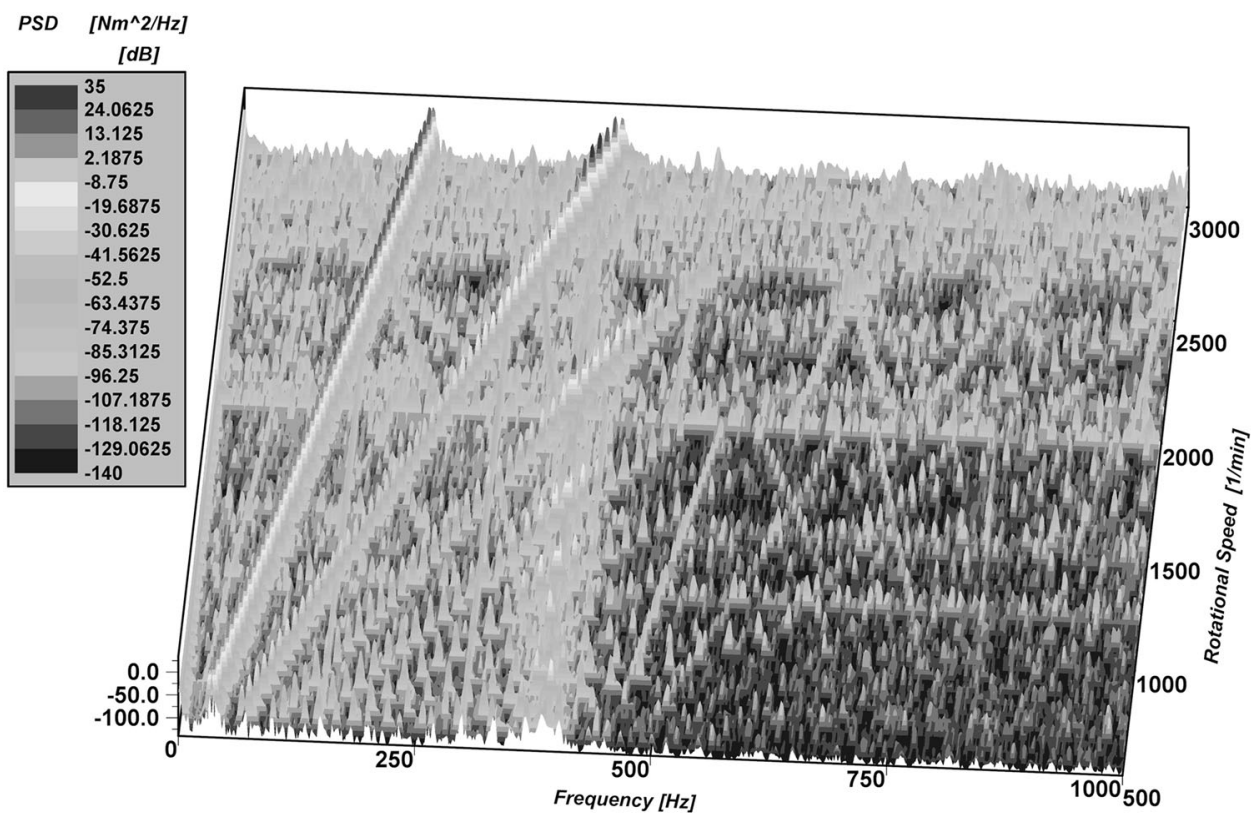

Moreover, vibration absorption is used to neutralize a certain resonance by adding a secondary system consisting of an auxiliary mass, spring and damping [11]. This approach is based on the torque of this subsystem being opposed to the excitation torque at the respective frequency. Another concept intervening in the modal behavior of the structure is represented by vibration isolation [10]. Generally, this approach is characterized by the separation of the response from the excitation in the frequency domain. Consequently, structural changes in mass, stiffness and damping generate a different modal behavior of the system as a whole and lead to the attenuation of the undesired output.

In the course of the analysis and discussion of the various vibration management possibilities, each method has to be evaluated with respect to quality, cost and time.

Energy dissipation by the addition of damping can improve the present issue. However, this approach solely represents a reduction of the consequences of the amplification due to resonance. The kinetic energy is converted to thermal energy through dissipation within rubber materials that exhibit a high damping ratio. This can lead to their structural damage and to the request of periodical damper swaps.

Furthermore, a cam lobe number which is not equal to the number of cylinders of the engine requires an intermediate shaft with an adequate transmission ratio. The pump cannot be driven by the camshaft anymore, as the number of pressure pulsations per revolution does not match the injection events. This impedes and deteriorates inevitably the control of the injected fuel mass.

Moreover, the use of a torsional absorber requires a complexly designed subsystem. It neutralizes the present 
Fig. 7 Schematic discussion of the modification possibilities of the driveline

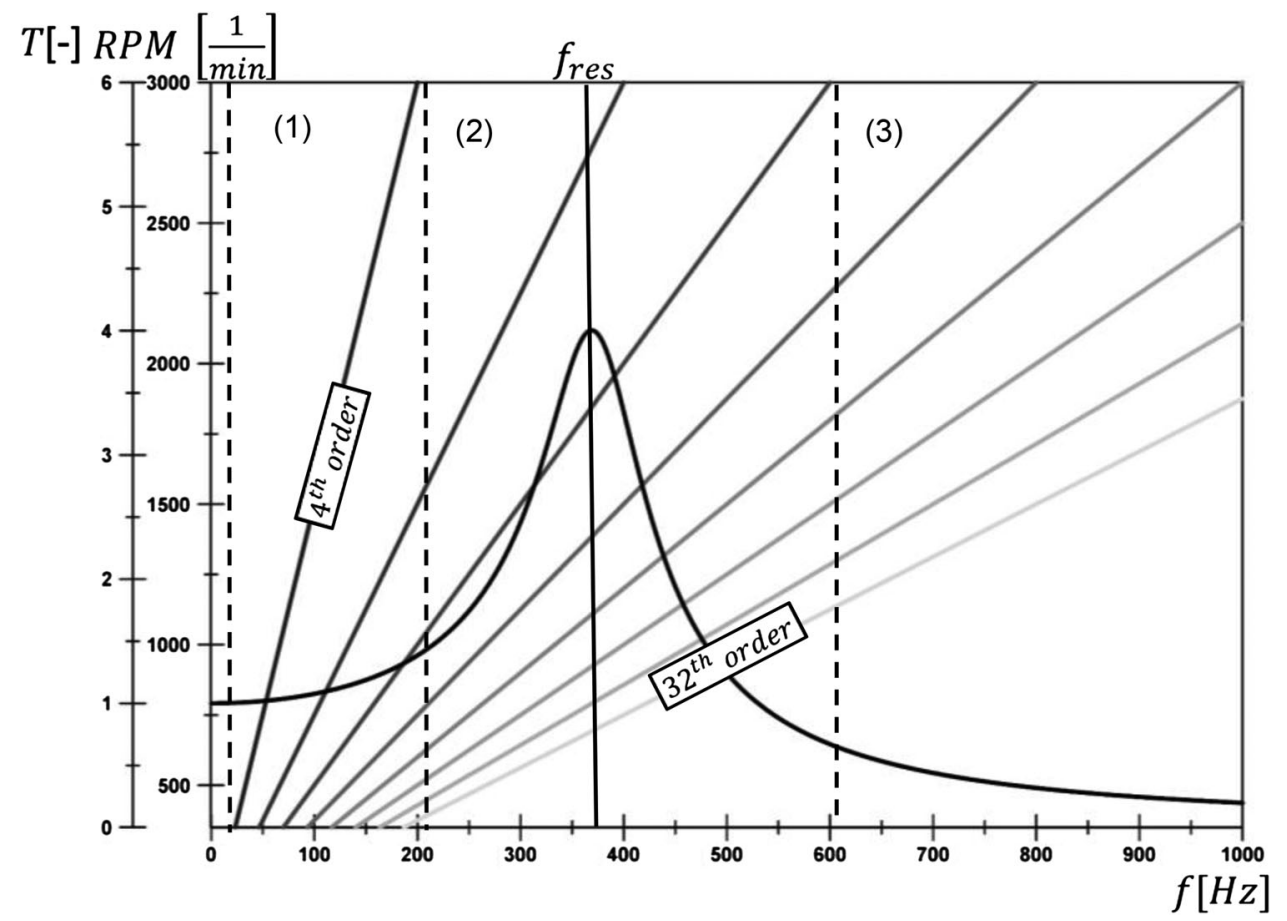

amplification. However, it is characterized by two new resonances if the original driveline is considered as a single degree of freedom (SDOF) system. One of these resonances is lower than the present eigenfrequency and therefore closer to the excitation provoking an undetermined response. Furthermore, the design of these vibration absorbers is complex and frequently characterized by iteration loops.

Prevalently, a comprehensive strategy for managing vibrations is the combination of several methods. This is due to the fact that the dynamic undesired torque proportions can be reduced and controlled, yet not eliminated.

All things considered, vibration isolation is the most adequate approach for an initial enhancement of the system to achieve better results concerning angular-resolved torque measurements, as it directly approaches the root cause of the measurement failure. However, separating the dynamic response from its excitation represents an exigent task in the present case, as part of the dynamic output is constituted by the signal to be measured and can be amplified or attenuated by the system's inherent vibrational characteristics. Since high pressure pumps of Otto engines are predominantly operated by cams with four or less lobes driven by the camshaft, the frequency range of the most influential 4th rotation order of the signal to be measured lies between $f_{4.0 \_ \text {low }}=23, \overline{3} \mathrm{~Hz}(350 \mathrm{RPM})$ and $f_{4.0 \_ \text {high }}=$ $200 \mathrm{~Hz}(3000 \mathrm{RPM})$ or lower. This frequency band should never be disturbed by any resonance of the modified system. Many modifications in terms of vibration isolation are based on the strategy of shifting the predominant resonance below the lower limit of the excitation band. In this way, the excitation and especially its higher harmonics do not cross $f_{\text {res }}$. Provided that higher eigenmodes are negligible, this strategy completely separates the dynamic input from its output, avoiding the system to resonate. In similar way, the first torsional eigenfrequency could be shifted slightly below the lower limit of the frequency band of the 4th order $f_{4.0 \_ \text {low }}$. This opportunity of changing the modal behavior of the driveline is depicted in the schematic Campbell plot in Fig. 7 and indicated by (1).

Yet, it requires a very high mass of inertia $J_{\text {eq }}$ combined with an overall soft torsional stiffness $c_{\text {eq }}$. Both effects restrain the agility of the system. Additionally, soft structures with high damping ratios lead to high thermal impact, especially in terms of component endurance testing or measurement programs with long durations. In a second approach, the torsional resonance could be downshifted slightly above $f_{4.0 \_ \text {high }}$, indicated by (2). Yet, the rotations per minute (RPM) band are very broad and consequently the signal would be deteriorated by the 8th order and higher harmonics for rotation speeds $n \approx 1500$ RPM and lower. Furthermore, if the observed SDOF transmissibility behavior is taken into account with an assumed maximum amplification at resonance of $T \approx 4$, the signal to be measured will be generally amplified, even if the rotation order does not equal resonance. Especially by downshifting the resonance, the amplification and the described effect increase. Additionally, downshifting the first torsional resonance also influences higher torsional eigenmodes. Although the behavior of the system is dominated by the 
Fig. 8 Outline of structural changes in driveline (C) Daimler AG)

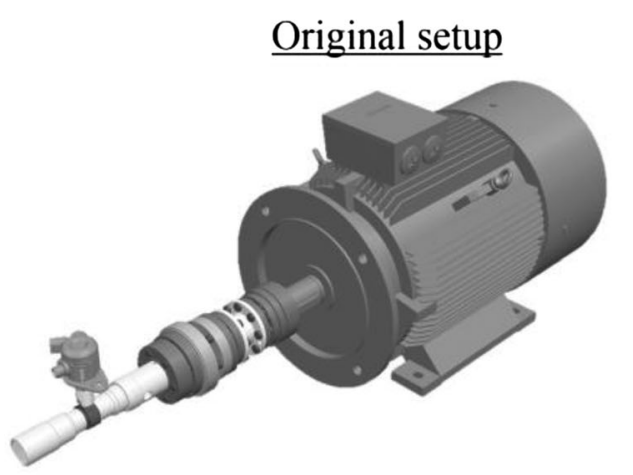

Modified setup

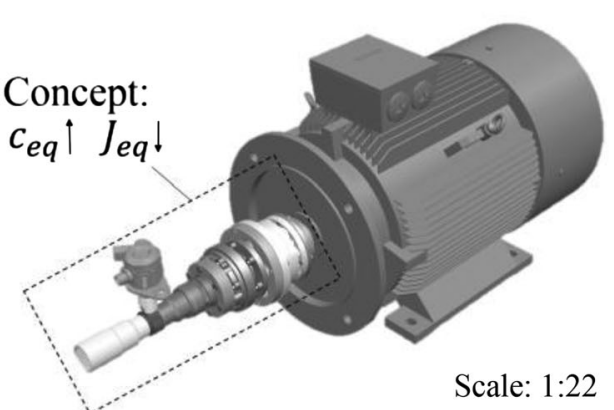

first eigenmode and behaves like a SDOF concerning the torque measurement for a frequency range $\leq 1 \mathrm{kHz}$, this could change by downshifting higher modes.

The transient behavior of the driveline evaluated in Fig. 5 shows, that the resonance is excited by multiples of this 4th order. Here, especially the 8th and the 12th order are predominant. All things considered, this yields a range from $f_{4.0 \_ \text {low }}=23, \overline{3} \mathrm{~Hz}$ up to $f_{12.0 \_ \text {high }}=600 \mathrm{~Hz}$ in which the signal can be deteriorated significantly. Taking the discussed modification possibilities into consideration, the overall torsional stiffness $c_{\text {eq }}$ of the driveline is raised and its overall moment of inertia $J_{\text {eq }}$ is reduced to shift the resonance above $f_{12.0}$ high to the highest possible frequency, indicated by (3) in Fig. 7. Hence, the system transmits the torque of the high pressure pump with a transmissibility $T \approx 1$ for low frequencies, according to the underlying SDOF principle. Moreover, it attenuates and falsifies the signal to be measured for inputs with frequencies higher than $\sqrt{2}$ times of $f_{\text {res }}$.
Design changes leading to this upshift of the first torsional resonance of the arrangement are combined and realized in a restructured driveline. In general, these objectives are achieved by a variation of geometry and a different choice of materials. Concerning material changes, the goals are combining low density and high elastic shear modulus to achieve an overall high torsional stiffness of the modified driveline. Hence, alternatives to the steel setup are found in combining aluminum with titanium elements. Geometrically, a shortened structure together with larger diameters of the rotating parts and partially hollow shaft bodies are realized. An outline of the modified driveline setup in assembled configuration is depicted in Fig. 8.

\subsection{Analysis and evaluation}

The enhanced system is evaluated by performing reference measurements at the same operating points both with the original and the improved setup. Figure 9 depicts the
Fig. 9 Campbell diagram of measured torque signal after structural changes (C) Daimler $\mathrm{AG})$

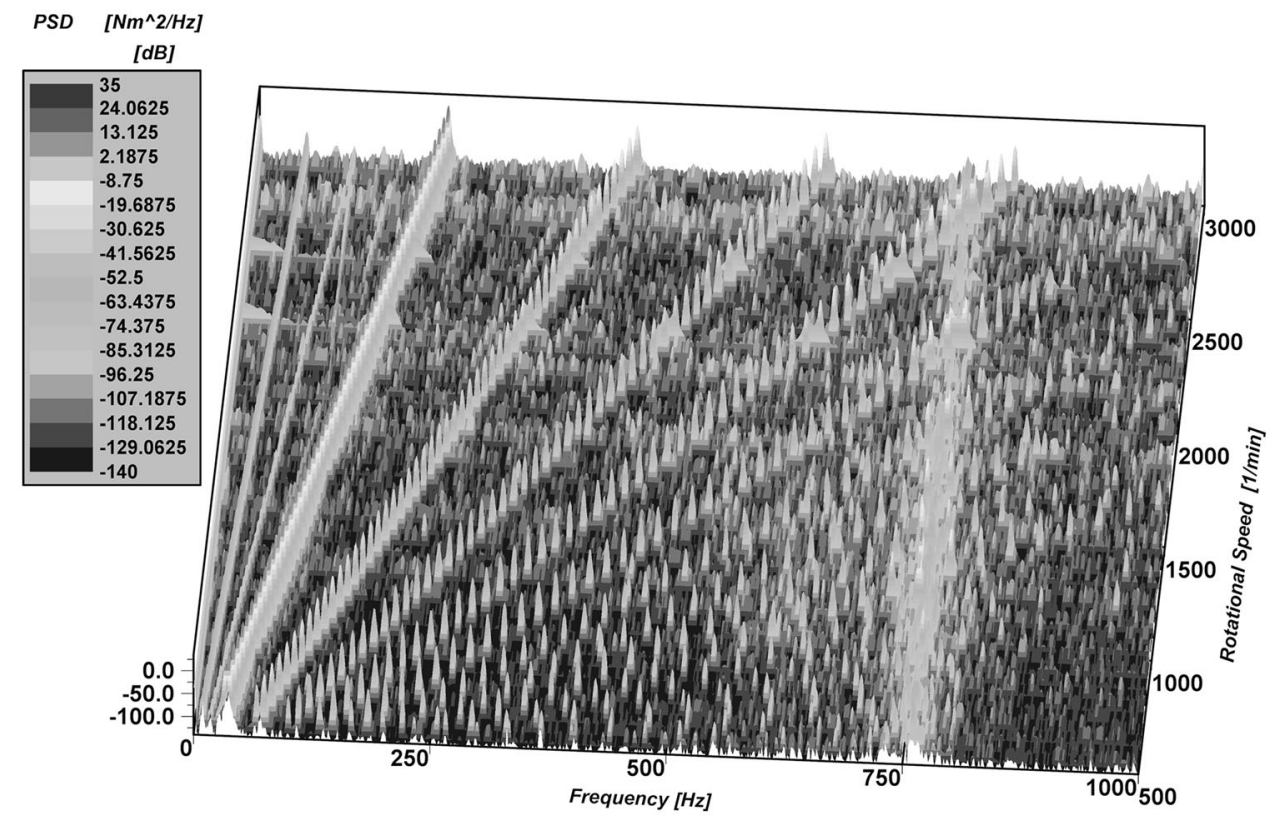


Fig. 10 Torque signal at 2700 RPM, 400 bar, $40 \mathrm{~kg} / \mathrm{h}$ after structural changes gray before modification; black after modification (@ Daimler AG)

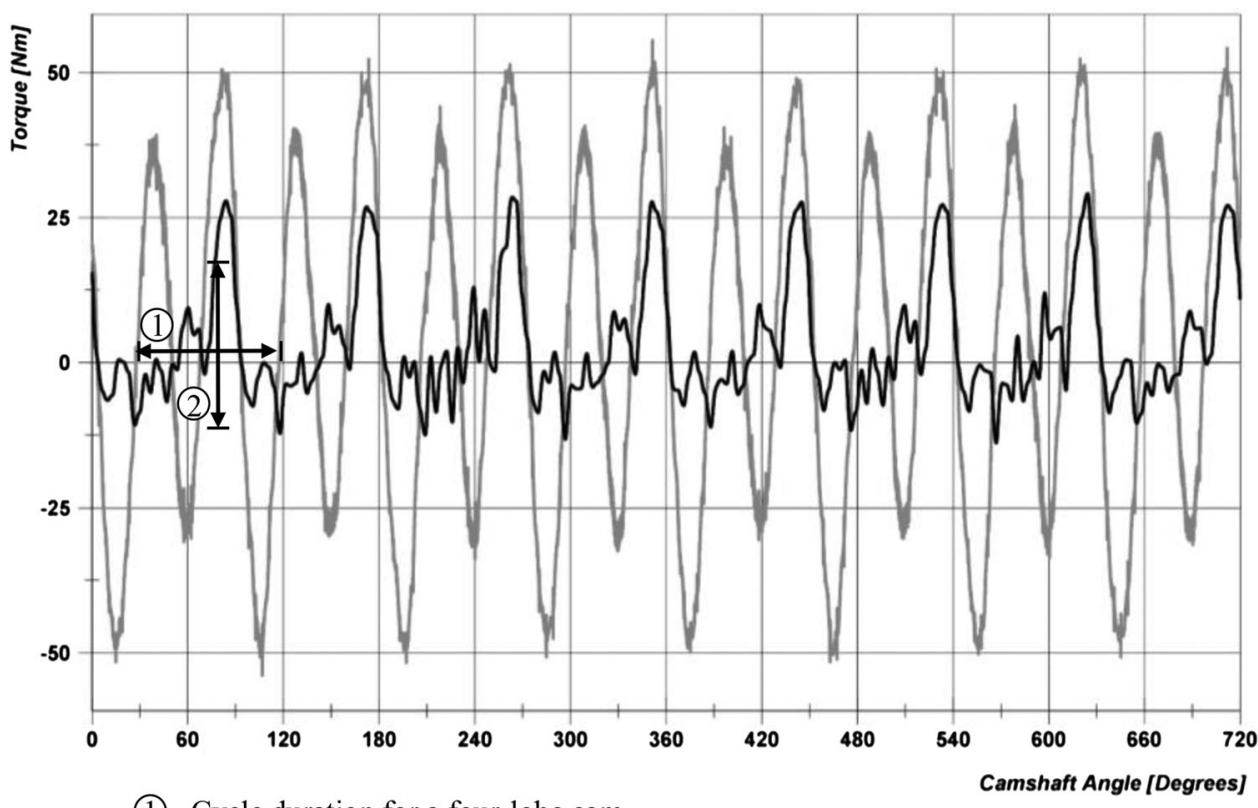

(1) Cycle duration for a four-lobe cam

(2) Mathematically estimated working stroke of the torque
Campbell diagram for the structurally modified test setup. The resonant frequency ascends to $\sim 750 \mathrm{~Hz}$, exposing the 8th and the 12th order of the signal to be measured entirely. In turn, the excitation of the resonance at constant rotation speed is driven by higher orders with lower amplitude. This leads to a significant overall reduction of the undesired dynamic proportion of the torque signal, as shown in Fig. 9. Besides that, the transmissibility of the rotation orders changes according to the assumed SDOF behavior. In contrast to the unmodified structure in Fig. 5, the new setup transmits the 4 th, 8 th and 12 th rotation orders of the torque pulsations with negligible amplification in the whole RPM band.

The influence on a certain tripe in the time domain is exemplarily stated in Fig. 10. Once more, the measured torque is depicted at the operating point of (2700 RPM, 400 bar, $30 \mathrm{~kg} / \mathrm{h}$ ) and compared to the problem statement in Fig. 3.

The modification of the driveline leads to the identification of the eight pump strokes over two revolutions. Moreover, the amplitude decreases, approximating the torque maximum of each lobe with a reasonable degree of accuracy compared to the simulation. It is obvious that the resonance of $\sim 750 \mathrm{~Hz}$ continues influencing the signal. Furthermore, some rotation speeds provoke resonance that did not before the modification and vice versa. However, the signal in its entirety is improved significantly due to a substantially reduced impact of the first torsional resonance.

\section{Summary and outlook}

This paper addresses torque measurements performed on a component test facility for high pressure fuel pumps. In particular, an undesired dynamic proportion in the unfiltered torque signal which is acquired by the use of a strain gage based sensing element in the driving shaft is focused. The facility, its mechanical, electrical and hydraulic branches are outlined and analyzed. A fault tree analysis is presented and applied to locate the origin of the signal failure. After identifying the first torsional driveline resonance as root cause, vibration management possibilities are considered and discussed. A first method of structural modification is realized and the signal is evaluated. As a result, the signal improves both in frequency and amplitude by implementing a concept based on the methodology of vibration isolation. Further enhancement can be realized, e.g., by combining the vibration isolation concept with a certain amount of additional damping or friction and energy dissipation within the driveline clutch. Another beneficial approach is represented by combining the structural changes with mathematical methods. Hence, the angular-resolved torque signal after modification can be complemented by using a Vold-Kalman order tracking filter for the exposed 4th, 8th and 12th rotation order. 


\section{References}

1. Vogel, F.: Wirkungsgradanalyse von Kraftstoffhochdruckpumpen. Bachelor Thesis, Ettlingen University of Applied Sciences (2012)

2. Triendl, T.: Einflussanalyse des Wirkungsgrads von DieselHochdruckpumpen auf den CO2-Ausstoß. Master Thesis, Technische Universität Wien (2013)

3. Schicker, R., Wegener, G: Drehmoment richtig messen. Hottinger Baldwin Messtechnik GmbH (2002)

4. Klaus, L.: Entwicklung eines primären Verfahrens zur Kalibrierung von Drehmomentaufnehmern mit dynamischer Anregung. Dissertation, Gottfried Wilhelm Leibniz Universität Hannover (2016)

5. Vesely, W.E., Goldberg, F.F., Roberts, N.H., Haasl, D.F.: Fault Tree Handbook. Government Printing Office, Washington, D.C. (1981)

6. Schwindt, E.: Gefahrenanalyse mittels Fehlerbaumanalyse. Universität Paderborn, Skriptum (2004)
7. Schwarz, B.J., Richardson, M.H.: Introduction to Operation Deflection Shapes. CSI Reliability Week, Orlando (1999)

8. Ropers, C., Saemann, E.-U., Gauterin, F.: Hybridmethode zur Bestimmung der Betriebsschwingungen von Reifen. In: Tagungsband der DAGA 03 (Fortschritte der Akustik), pp. 302-303. (2003)

9. Heiman, B., Popp, K.: Maschinendynamik Vorlesungsskript. Institutsverbund für Mechanik der Universität Hannover, Hannover (2005)

10. Rao, S.S.: Mechanical Vibrations, 6th edn. Pearson, New York (2017)

11. Mirsanei, R., Hajikhani, A., Peykari, B., Hamedi, J.: Developing a new design for adaptive tuned dynamic vibration absorber (ATDVA) based on smart slider-crank mechanism to control of undesirable vibrations. Int. J. Mech. Eng. Mechatron. 1(1), 80-87 (2012) 